\title{
UN MODELO DE RECONFIGURACIÓN DEL TERRITORIO: LA EUROCIUDAD CHAVES-VERÍN Y LA COOPERACIÓN TURÍSTICA EN EL ALTO TÁMEGA*
}

\author{
Xulio Pardellas de Blas \\ Carmen Padín Fabeiro \\ Facultad de Economía. Universidad de Vigo \\ xulio@uvigo.es, padin@uvigo.es
}

\section{RESUMEN}

La cooperación entre ciudades constituye un nuevo enfoque de la gestión turística en destinos urbanos, dada la complementariedad de muchos de los productos ofertados. El caso que se presenta es el de dos ciudades, Chaves y Verín, que perteneciendo a países diferentes, tienen sólidos vínculos históricos de intercambio y cooperación y un largo recorrido de relaciones sociales. La oportunidad abierta por su incorporación al Eixo Atlántico y la nueva programación comunitaria 2007-2013, han permitido acelerar el proceso de construcción de una eurociudad, donde el turismo puede jugar un papel relevante en el nuevo modelo territorial.

Palabras clave: destino turístico, cooperación transfronteriza, turismo urbano.

\section{ABSTRACT}

The cooperation between cities is a new focus on tourism management, taken account of complementary of much of its offers. The case of this study is about two cities, Chaves and Verin, who own to different countries but with solid historic links of interchange and cooperation and a long way in its social relationships. The opportunity opened by its engaged to the Eixo Atlántico and the new UE programme 2007-2013, has permitted to hurry the construction process of an eurocity, when tourism can give a relevant role in the new territorial model.

Key words: tourism destination, border cooperation, urban tourism.

Fecha de recepción: mayo 2011.

Fecha de aceptación: diciembre 2012.

* Con financiación del Ministerio de Ciencia e Innovación. Referencia: CSO2011-29529-C04-03”. 


\section{INTRODUCCIÓN Y METODOLOGÍA}

La cooperación territorial dentro de la Unión Europea y, concretamente la transfronteriza, están entrando en la segunda generación, o más exactamente, pasando de la fase del predominio de las infraestructuras a la fase de la preponderancia del vivir de los ciudadanos, artificialmente separados. Esta es la apuesta comunitaria: la cooperación de cercanías. En este contexto, las Eurociudades son elementos muy importantes, entendidas como auténticos laboratorios de construcción de ciudadanía y experimentación de vivencias compartidas entre iguales.

Chaves y Verín son dos ciudades con sólidos vínculos históricos de intercambio y cooperación y la idea de crear estructuras institucionales para fortalecer las relaciones entre ambas tiene un largo recorrido de relaciones entre las dos instituciones locales, desde hace algún tiempo. No obstante, fue la oportunidad abierta por el impacto de dos nuevos factores quien permitió la puesta en marcha del actual proceso de construcción de una Eurociudad: la incorporación de las dos ciudades al Eixo Atlántico -asociación de municipios de la eurorregión que potencia la cooperación transfronteriza a nivel local-, y la nueva programación comunitaria 2007-2013, con su especial hincapié en la cooperación territorial, elevada a objetivo principal, junto con la convergencia y la apuesta por el crecimiento y el empleo.

Aún siendo una iniciativa plenamente local, nacida con un fuerte impulso social, después de su integración en 2005 en el Eixo Atlántico, el proyecto recibió un fuerte apoyo porque coincidía plenamente con las orientaciones estratégicas trazadas para el período 2007-2013 en la Agenda Estratégica del Eixo, que tiene como pilar básico justamente, el fomento de la gobernanza y de estructuras de cooperación, objetivos fundamentales del concepto de Eurociudad. De ese modo, en diciembre de 2007, los dos alcaldes presentaron oficialmente en Chaves el proyecto de Eurociudad, apadrinado por el Secretario de Estado de Desenvolvimento Regional de Portugal, por el Conselleiro de Economía de la Xunta de Galicia, por el Vicepresidente de la Comisão de Coordenação e Desenvolvimento da Região Norte (CCDR-N) y por el Presidente de la Diputación de Ourense, entre otras autoridades. Una de las primeras decisiones fue el encargo a un grupo de profesores e investigadores de las Universidades de Vigo y Trás-Os-Montes, para elaborar un diagnóstico y unas propuestas de actuación, que fueron presentadas a finales de 2009 y que en parte se resumen en este trabajo.

La opción metodológica elegida partió de una realidad que reconocía a la eurociudad como un proceso ya en marcha, con una relativamente elevada aceptación social, lo que significaba una escala específica de análisis con indicadores de geometría variable y en consecuencia, la búsqueda de información no podía limitarse a documentos escritos (muy escasos), sino extenderse a los actores sociales involucrados en el proceso. Desde finales de 2008 y a lo largo de la primera mitad de 2009, se llevaron a cabo encuentros interactivos y entrevistas en profundidad con representantes institucionales, con agentes sociales y económicos, y asimismo, con delegados de los medios de comunicación, que en un total de 60 personas, aportaron datos, opiniones y debates sobre el concepto de eurociudad y su posible evolución, y sobre las actuaciones necesarias para llevar aquella idea a buen fin. 


\section{REVISIÓN DE LA LITERATURA CIENTÍFICA}

A principios de los años 80 se produce la confluencia de dos líneas de investigación que darán lugar a lo que se conoce como desarrollo endógeno: una de carácter teórico, que nace como consecuencia de la tentativa de encontrar una noción conceptual que permitiera la acción pública para el desarrollo de localidades y regiones, y otra de carácter empírico, que surge derivada de la interpretación de los procesos de crecimiento industrial en lugares concretos del sur de Europa (Garofoli, 1983; Vázquez Barquero, 1983).

Los procesos de industrialización endógena aparecen firmemente enraizados en el territorio, surgieron en pequeñas y medianas ciudades de la mano de los emprendedores locales, y a lo largo del tiempo fueron acumulando un saber técnico, formaron sistemas relacionales propios y consolidaron formas culturales autónomas que explican su continuidad en el tiempo. Así fue creándose un entorno económico e institucional que proporciona a las empresas locales recursos, servicios y redes de cooperación que les permiten mejorar su competitividad en los mercados (Aydalot 1986).

Con este enfoque, el territorio pasó a ocupar un papel de agente de transformación dejando de ser un simple soporte de los recursos y de las actividades económicas, ya que las empresas y demás actores del territorio interactúan entre sí, organizándose para desarrollar la economía y la sociedad. El punto de partida del desarrollo en la comunidad territorial es el conjunto de recursos (económicos, humanos. institucionales y culturales) que constituyen su potencial endógeno. Son justamente las pequeñas y medianas empresas las que, con su flexibilidad y su capacidad emprendedora y organizativa están llamadas a tener un papel protagonista en los procesos de desarrollo endógeno (Vázquez Barquero, 2005).

Aplicando este concepto a los casos de Galicia y Norte de Portugal, se observa que el impulso de inicio de los cambios estructurales en su sistema productivo en los años 80 después de la crisis, parece responder a este modelo. La iniciativa empresarial contó con una importante dotación del recurso trabajo, con la mano de obra que se incorporó a la producción industrial procedente del sector agrícola, igualmente fue utilizada la disponibilidad del ahorro procedente de la misma actividad agraria o comercial, y singularmente con las remesas de la emigración, y sobre todo, encontró una estructura territorial consolidada y un fuerte apoyo social e institucional (Comission Europènne, 1999).

El desarrollo endógeno en la eurorregión constituye así un proceso que tomó sentido en el territorio y fue articulándose sobre el entorno local de las ciudades, donde se concentraban las externalidades positivas producidas por la dinámica conjunta de los actores sociales y agregadamente, por la creación institucional de condiciones favorables para el comercio y la actividad económica, lo que provocó una reducción de los costes de transacción entre las empresas y el resto de los agentes económicos de las dos regiones. En este contexto, el papel de las infraestructuras fue claramente definidor de un modelo de cooperación que además de reconfigurar el territorio ya en el corto plazo, puede reubicarlo en los mercados europeos y mundiales, aún a pesar de su situación periférica en términos geográficos y económicos (Pardellas, 2009).

Desde esa misma filosofía, el actual contexto económico, caracterizado por los continuos cambios tecnológicos, incertidumbre de los mercados y elevada competitividad en todos los sectores, provocó nuevos desafíos para los sistemas económicos y para las empresas, que 
deben desarrollar nuevos productos y a la vez diferenciarse de los competidores a todos los niveles. En este marco de actuación la innovación y la cooperación desempeñan un papel fundamental en la competitividad, ampliando este término a los territorios. Ahora bien, la aplicación del concepto de competitividad a la problemática territorial, tanto urbana como regional, presentó en los últimos años serias dificultades metodológicas, considerando dos planos diferentes de enfoque: el microeconómico, circunscrito a los problemas empresariales y el macroeconómico, desde la óptica regional o nacional, que serán estudiados más adelante.

En general, desde el enfoque micro existe cierto consenso sobre el concepto y los instrumentos e indicadores, lo que permite realizar comparaciones a nivel internacional. Aún así, desde el ámbito de la Unión Europea viene suscitándose la necesidad de incrementar la competitividad regional con los programas integrados en los Fondos Estructurales, que buscan acentuar los esfuerzos para conseguir territorios equilibrados, con unos niveles de competitividad similares. En ese sentido la competitividad aparece asociada, en el enfoque macroeconómico, a la capacidad de una economía para transferir su producción a actividades con mayor productividad y que deben generar un nivel más elevado de salarios reales. La competitividad debe analizarse por lo tanto, asociada a niveles crecientes de vida y mayores oportunidades de empleo, no solamente en cantidad sino en calidad.

Además, si la aplicación del concepto de competitividad a la problemática territorial (competitividad regional y urbana), se enfrenta a aquellas dificultades, no resultan menores para concretarla en un territorio donde confluyen por una parte las diferencias legislativas de dos países y por otra, las no menos importantes desigualdades en las competencias administrativas de la autonomía gallega y la, de momento inexistente, administración de la Região Norte. En este caso, operar con aquellas variables requiere necesariamente un difícil equilibrio entre el nivel micro (la calidad en la gestión de las empresas) y el nivel macro e institucional (las políticas de desarrollo de la Xunta de Galicia y de Lisboa (Figueiredo y Pardellas 2005).

Una buena ilustración global de estos problemas puede ser la notable aportación que el número de diciembre de 2004 de la revista Regional Studies, dedica al tema de la competitividad. El título del artículo introductorio de la revista, «Regional Competitiveness: an elusive yet key concept» (Kitson et al., 2004, citado en Figueiredo y Pardellas 2005), evidencia esa situación de paradoja, no dudando los autores en calificar el tema de la competitividad como una cuestión de fe, con innumerables fieles y seguidores, aunque el concepto esté lejos de ser inequívoco o exento de conflictividad.

Krugman $(1994,1996)$ fue de los primeros economistas en cuestionar la ligereza con que el concepto de competitividad es transportado de la realidad mercado - empresa (donde no ser competitivo implica desaparecer), hacia la idea de competitividad de la nación (Porter, 1990; 2002). Esta última vertiente experimentó significativos desarrollos conceptuales y metodológicos operados en el ámbito de la competitividad de las naciones, cruzando los trabajos pioneros de Porter con los del World Economic Forum, enriquecidos recientemente con aportaciones de economistas prestigiosos de la teoría del crecimiento económico (Sala i Martin, 2003).

Por otro lado, los documentos de la D. G. Regio sobre la programación de Fondos Estructurales para el período 2007-2013, acentúan fuertemente la vertiente de la competitividad regional, en cuanto prioridad de envolver un conjunto más significativo de territorios en los 
esfuerzos de producción sostenible de riqueza. En idéntico sentido, la centralidad de los indicadores de productividad en el concepto general introduce por sí mismo un fuerte conflicto, dado que nos lleva a la vasta literatura de los factores o determinantes del crecimiento de la productividad y en consecuencia, al debate hoy abierto entre las corrientes del crecimiento endógeno y del condicionado (Vázquez Barquero, 1999).

Tal y como otras aportaciones vienen a reconocer (R. Camagni, 2002; ECORYS, 2003), la evolución del concepto de competitividad regional surge así, fuertemente influenciado por dos líneas de progresión: la que parte de la competitividad de las empresas y su agregación, y la que tiende a prolongar el análisis de los determinantes macro de la productividad y su crecimiento hacia las regiones, por lo que es cada vez más necesario encontrar alguna perspectiva de síntesis entre las dos líneas de investigación.

Igualmente, deben ser considerados relevantes otros interesantes puntos de vista: el de Begg (1999), señalando que los factores que influencian la competitividad urbana pueden expresar también condiciones económicas nacionales y hasta internacionales, que trascienden el efecto directo de la propia dinámica de la ciudad. También, de forma complementaria, el de Turok (2004), recordando que las ciudades son, simultáneamente, fuente de ventajas (la dimensión de sus recursos y el papel como catalizadoras de su uso eficiente), y de problemas (fenómenos de desurbanización y de externalidades negativas, asociadas a los costes de aglomeración). Asimismo, Fernández Tabales et al (2009), aporta un interesante debate sobre los conceptos de cohesión y equidad territorial, que en última instancia implicarían una tendencia de la comunidad social a identificarse con un proyecto de vida común, indicador de singular relevancia para el caso que nos ocupa.

El debate científico en torno a la competitividad territorial apenas está iniciado, pero resulta evidente que un proyecto como el que nos ocupa, construcción de una Eurociudad, ha de inscribirse en esa línea para enfrentar los desafíos en el corto plazo. Quizás el equilibrio para compensar la asimetría de las competencias administrativas entre cada ribera del río Miño (la frontera tradicional por extensión, natural y administrativa entre Galicia y Portugal hasta su integración en la UE), podría estar garantizado en un principio a corto plazo, teniendo en cuenta el citado apoyo de la Xunta de Galicia y de la CCDR-N, lo que afectaría a las decisiones macro. Otro tema serán las decisiones micro, que inexcusablemente deben tomar las empresas y el resto de los actores sociales, pero tanto el diagnóstico, como las propuestas analizadas en este documento, tienen como objetivo acercar claves de interpretación y actuación que faciliten aquellas decisiones (González y Figueroa, 2006, 2007, 2008).

El otro enfoque complementario se orienta al análisis y configuración del espacio geográfico de los dos municipios de la Eurociudad como destino turístico, partiendo de una situación casi marginal en ese ámbito de la actividad económica, a pesar de la existencia de importantes recursos en su territorio, tanto en su patrimonio natural, como histórico, como asimismo, en sus singulares y recursos termales compartidos.

Probablemente los estudios de mayor relevancia para aquel análisis son los de Gunn (1972, 1993), alrededor del concepto de planificación regional, proponiendo un espacio destino con cinco elementos básicos: la definición precisa de los límites territoriales, un acceso desde los mercados con corredores de circulación interna, unos complejos de atracción, un hinterland no atractivo y entradas a la región. También desde la perspectiva del territorio municipal es muy interesante el análisis de Pearce (1995), que sintetiza varios trabajos de 
planificación y análisis desde la perspectiva de la evolución de los destinos, entre los que destacan el modelo de evolución espacio-temporal de Gormson (1981 in Pearce, cit.), que incorpora los cambios en el grado de participación local, y con mucho mayor interés para este estudio, los estudios de Opperman (1993) sobre el papel de los turistas informales para abrir un espacio de destino a los mercados «formales», y de Leiper (1995) que identifica las características y condiciones para que un destino sea atractivo.

Es cierto que la mayoría de los análisis citados se refieren a casos concretos, por ejemplo, los modelos que se ocupan de las pautas de comportamiento o los viajes multidestino se han desarrollado principalmente sobre datos de Norteamérica y tienen una utilidad limitada para planificar regiones de Europa, donde los factores espaciales y la vecindad de destinos con características físicas semejantes deben tenerse muy en cuenta para identificar con precisión un destino. Desde otra visión, el análisis de los instrumentos funcionales y evolutivos se han desarrollado con datos de estudios empíricos, tratando de explicar la comprensión de las características de un destino y aspectos de su desarrollo, o también los comportamientos de los turistas, lo que proporciona a las administraciones una importante información previa a su trabajo, pero le ayuda poco en la identificación de preferencias en función de objetivos territoriales.

Sin embargo, a pesar de esas limitaciones, podemos destacar ideas interesantes en esos modelos. Sin duda las de mayor relevancia aparecen en las aportaciones de Gunn (cit.) que contribuyen a la descripción de la estructura física de los territorios destino. Sus conclusiones son ampliamente citadas, siendo uno de los pocos que se ha centrado en la descripción y desarrollo de modelos estructurales de destinos turísticos. En su concepto más elaborado de planificación territorial, Gunn (1993) identifica cinco conceptos clave: frontera, acceso y corredor interno, complejos de atracción, áreas vecinas no atractivas y entradas. Desde otros enfoques, y casi simultáneamente, los citados Lue, Crompton y Fesenmaier (1993) identifican cinco modelos de viajes, destacando el modelo de viaje encadenado hacia «focos» de atracción.

Coincidiendo con ese análisis, Leiper (cit.) identifica tres componentes de un sistema de atracción: un núcleo, un turista y un marcador. El núcleo es el elemento central de las atracciones turísticas, el turista es quien viaja y mantiene un contacto personal con los lugares visitados y el marcador es un instrumento de información para el turista. En una línea próxima, Dredge (1999) aporta una interesante base teórica, que sintetiza y reestructura los anteriores, en especial las ideas de Gunn y Leiper, con una propuesta en la que destacan tres supuestos fundamentales como base de su modelo: que los mercados emisores de turismo y los territorios destino sean entidades geográficas separadas, que la naturaleza compleja y de distintos niveles de los destinos requiera una estructura jerárquica pero flexible, y que el modelo de planificación y diseño comprenda un espacio de destino, mercados emisores de turismo, nodos, distritos, rutas circulatorias y puertas (1999: 403). Por su parte, los estudios más recientes de Swarbrooke y Horner (1999) y de Pizam y Mansfeld (2000), contrastan el modelo de comportamiento de los turistas con respecto a destinos donde existan o puedan crearse nodos de atracciones y estructuras de recorrido circular, y desde un enfoque multidisciplinar, el trabajo dirigido por Bigné y López (2000) aporta varias reflexiones sobre la calidad y la competitividad relacionadas con la necesidad de diseñar el desarrollo de destinos turísticos. Finalmente, en el ámbito conceptual del turismo transfronterizo, la aportación 
de Cuevas y Zizaldra (2009), presenta relevantes resultados sobre las redes de empresas de notable importancia para esta investigación.

Teniendo en cuenta las circunstancias del proceso de construcción de la Eurociudad, parece posible analizar el papel del turismo como un factor relevante en su desarrollo utilizando las citadas aportaciones de Lue, Crompton y Fesenmaier sobre los viajes multidestino, y de Leiper sobre los elementos de atracción, así como la propuesta de Dredge, que permite su aplicación concreta a un espacio de destino, configurando desde el enfoque de la demanda una base importante para predecir el comportamiento de los turistas, proponiendo una oferta circular sobre la base de los recursos termales, con estancias alternas y visitas sucesivas, no sólo al territorio conjunto de los dos municipios, sino a toda el área geográfica circundante que formó en su día la frontera administrativa entre los dos países.

\section{EL ESPACIO DE ESTUDIO: COOPERACIÓN Y MERCADO TURÍSTICO}

Chaves es un municipio con cerca de 45.000 habitantes (de los cuales cerca de 20.000 en el perímetro urbano), siendo el casco urbano estructurante del territorio del Alto Tâmega (6 municipios con cerca de 100.000 habitantes). Verín, por su lado, es un municipio con cerca de 13.000 habitantes, centro de una extensa área funcional (que incluye a la Comarca de Monterrei-Verín, con más de 35.000 habitantes y también algunos municipios en comarcas vecinas).

Las dos ciudades distan entre sí unos $30 \mathrm{kms}$ por la antigua carretera (algo más por la nueva autovía que las une) y aunque dentro de la periferia peninsular, aparecen bien encuadradas en el espacio ibérico formando parte de la «Y» griega que acerca la meseta a las dos principales ciudades costeras de la eurorregión: a través de la autovía portuguesa A7 de enlace al Área Metropolitana de Porto y de la española A52 de acceso al área de Vigo. Otros factores de centralidad funcional refuerzan esa situación, el Parque de Actividades de Chaves y la Plataforma Logística Transfronteriza Chaves-Verín (integrada en el Programa Portugal Logístico), son ya realidad concreta de una futura plataforma logística multicentros y multifuncional, prevista en el Plan Estratégico Ourense 2010-2015 (Consellería de Presidencia. Xunta de Galicia, 2010).

Observando los antecedentes de la cooperación entre las dos ciudades, podemos afirmar que está ya anclada en diversas dinámicas convergentes (Domínguez, 2008):

- La cercanía geográfica, histórica, cultural y político-institucional, que fue reforzada en los últimos años, a través de iniciativas previas de cooperación transfronteriza, de mejoras en la accesibilidad y movilidad, y en la consolidación de un espacio de vida común (residencia, ocio, empleo)

- La existencia de factores de complementariedad comunes, como pueden ser recursos territoriales (el valle del Támega, las aguas termales), económicos (la citada logística empresarial), culturales (idioma, música), y problemas de gestión urbana y territorial igualmente comunes

- Proyectos comunitarios conjuntos, como fueron dentro de Interreg III, dos centros de interpretación museística, la promoción de una red cultural o el itinerario fronterizo del Contrabando 
- Un intercambio ya experimentado en los ámbitos de la educación y la salud, que implicaba por un lado estancias y docencia compartida para alumnos de bachillerato, y por otro lado, la atención sanitaria en los hospitales de las dos ciudades en función de necesidades no cubiertas.

En este contexto, la creación de la Eurociudad figura como una apuesta ambiciosa y coherente con el pasado reciente, reuniendo dos características muy importantes para convertirse en una singular experiencia europea de este tipo. En primer lugar, la voluntad política de las administraciones (locales, provinciales, autonómicas y nacionales) para llevar adelante la iniciativa. En segundo lugar, su condición de puerta de acceso al Eje interior de la eurorregión Galicia-Norte de Portugal, un espacio necesitado de medidas de apoyo por su carácter de territorio de baja densidad demográfica y escaso dinamismo económico, que con actuaciones de este tipo mejorarían su competitividad y sus oportunidades de convergencia con el Eje litoral.

\section{El mercado turístico en Chaves y Verín}

La oferta turística de las dos ciudades hasta 2009 podía considerarse como un factor claramente marginal en su economía y a pesar de la existencia de notables y singulares recursos, un elevado número de ellos permanecen todavía inexplotados o infrautilizados. Entre todos ellos obviamente, destaca la riqueza en aguas termales y mineromedicinales, gracias a una misma falla geológica norte-sur, desde Verín hasta Peço da Régua, en la orilla del Douro (Domínguez 2008. cit).

El otro y no menos importante recurso hídrico, es el propio río Támega, que nacido en la Sierra de San Mamede, se abre paso por el valle de Monterrei, atravesando Verín y recorriendo el distrito de Chaves, hasta fundirse con el Douro en la localidad portuguesa de Entre-Os-Ríos. Una de las solicitudes que contempla el proyecto de eurociudad es su declaración como LIC en todo el recorrido, dado que hoy solo está reconocido el tramo que discurre por territorio gallego.

La arquitectura militar es consecuente con la historia de ambos países en frecuente conflicto bélico, de la que se conservan las fortalezas medievales de Monterrei (a escasa distancia de Verín) y la de Chaves, en el centro urbano, convertida una parte en la actualidad en hotel de 4*. Más recientes en el tiempo son los castillos de Aguiar y Montalegre en el lado gallego y Sto Estevão, Monforte e Mão Vicinho en el distrito de Chaves.

El uso turístico de los recursos complementa la naturaleza, la historia y la etnografía, existiendo ya, aunque con un potencial de elaboración y promoción mucho mayor que la presentación actual, la Ruta del Oro y las Vistas Mágicas, la de los Embalses (Barragems), la del Arte Rupestre y la del Contrabando, además de una ramificación del Camino de Santiago, que sube desde el Algarve, paralelo a la frontera con Castilla y muy utilizado por los conversos mozárabes en los s. XVI y XVII.

Las fiestas y la gastronomía son igualmente recursos singulares en este territorio, destacando dos de los carnavales más ancestrales de Galicia en Laza y Verín, y desde luego, la presencia de vinos de calidad, amparados por la Denominación de Origen Monterrei, en el caso gallego y por la indicación geográfica VQPRD (Vinhos de Qualidade Produzidos en 


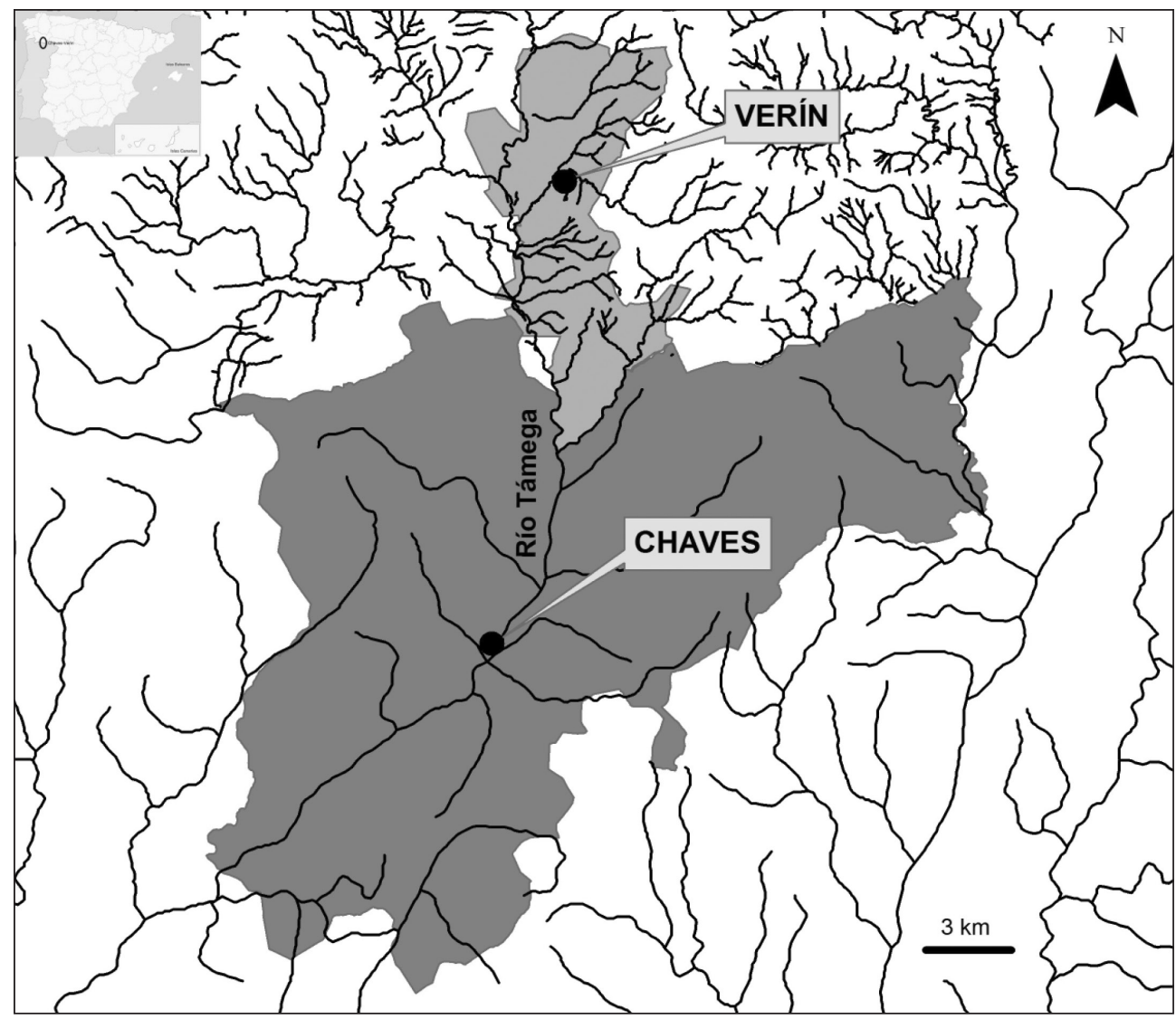

Fuente: Elaboración propia a partir de: Sistema Nacional de Informação de Recursos Hídricos (www.snirh.pt), Instituto Geográfico Portugués (http://www.igeo.pt/), SITGA (www.sitga.xunta.es) y Sistema integrado de información del agua (http://servicios2.marm.es/sia/visualizacion/descargas/documentos.jsp)

uma Região Determinada), concretamente, el Vinho Verde en Ribeira de Pena, el Vinho de Valpaços y el Vinho Maduro de Chaves, en el lado portugués (CCDRN 2007).

Con todo, la oferta de alojamiento muestra una estructura muy asimétrica en los dos municipios, tal como aparece en la tabla 1, donde observamos que las plazas hoteleras en Chaves casi triplican a las de Verín, lo que puede explicarse por el mayor peso de la actividad termal tradicional en el municipio portugués. En todo caso, es evidente que la mayoría de las plazas corresponden a establecimientos de muy baja categoría en Verín y media-baja en Chaves, lo que constituye una importante barrera, como se explicará más adelante, para elaborar una oferta turística de calidad dentro del proyecto de eurociudad.

Con respecto a la demanda, no existen datos cuantitativos desagregados para los dos municipios, pero un estudio realizado por los autores en 2008 sobre la actividad turística en 
Cuadro 1

OFERTA DE ALOJAMIENTO EN CHAVES Y VERÍN

\begin{tabular}{|c|c|c|c|c|}
\hline & Chaves & Verín & Chaves & Verín \\
\hline Alojamiento & \multicolumn{2}{|c|}{$\mathrm{n}^{\mathrm{o}}$} & \multicolumn{2}{|c|}{ Plazas } \\
\hline Hotel 4*/ 3* & 4 & 2 & 596 & 128 \\
\hline Hotel 2* & 1 & 1 & 78 & 60 \\
\hline Hotel 1* & - & 2 & - & 98 \\
\hline Hostal 1* & - & 5 & - & 146 \\
\hline Albergaria 4* & 1 & - & 107 & - \\
\hline Pensión & 9 & 4 & 309 & 96 \\
\hline Motel & 1 & - & 60 & - \\
\hline Turismo Espacio Rural (TER) & 14 & 0 & 195 & 0 \\
\hline Total & 30 & 14 & 1345 & 528 \\
\hline
\end{tabular}

Fuente: Elaboración propia a partir de datos de www.turgalicia.es , www.verin.net

las ciudades del Eixo Atlántico (Pardellas, 2009), revelaba algunos rasgos de las características y motivaciones de los turistas que acuden a estas dos ciudades. Los datos proceden de una encuesta realizada en los puntos de información turística local sobre 200 visitantes en cada ciudad, en la primavera y los meses de verano de 2008.

Cuadro 2

RASGOS DE LADEMANDA TURÍSTICA EN CHAVES-VERÍN

\begin{tabular}{|l|l|l|l|l|l|l|l|l|l|l|l|}
\hline Ch & Ve & Ch & Ve & Ch & Ve & Ch & Ve & Ch & Ve & Ch & Ve \\
\hline
\end{tabular}

\begin{tabular}{|l|l|l|l|l|l|}
\hline \multicolumn{2}{|c|}{$<\mathbf{3 0}$} & \multicolumn{2}{c|}{$\mathbf{3 0 - 6 0}$} & \multicolumn{2}{c|}{$>\mathbf{6 1}$} \\
\hline $24 \%$ & $8 \%$ & $63 \%$ & $76 \%$ & $14 \%$ & $16 \%$ \\
\hline
\end{tabular}

\begin{tabular}{|c|c|c|c|c|c|c|c|c|c|}
\hline \multicolumn{2}{|c|}{ Galicia } & \multicolumn{2}{c|}{ Portugal } & \multicolumn{2}{c|}{ España } & \multicolumn{2}{c|}{ Europa } & \multicolumn{2}{c|}{ América } \\
\hline $1 \%$ & $6 \%$ & $97 \%$ & $0 \%$ & $0 \%$ & $87 \%$ & $2 \%$ & $5 \%$ & $0 \%$ & $2 \%$ \\
\hline
\end{tabular}

\begin{tabular}{|c|c|c|c|c|c|c|c|c|c|c|c|}
\hline \multicolumn{2}{|c|}{ Folletos } & \multicolumn{2}{|c|}{ Rec. amigos } & \multicolumn{2}{c|}{ Agencias } & \multicolumn{2}{c|}{ Visit. anterior } & \multicolumn{2}{c|}{ Internet } & \multicolumn{2}{c|}{ Otros } \\
\hline $15 \%$ & $7 \%$ & $32 \%$ & $72 \%$ & $3 \%$ & $2 \%$ & $17 \%$ & $3 \%$ & $25 \%$ & $8 \%$ & $8 \%$ & $8 \%$ \\
\hline
\end{tabular}

\begin{tabular}{|c|c|c|c|c|c|c|c|c|c|c|c|}
\hline \multicolumn{2}{|c|}{ Patrimonio } & \multicolumn{2}{|c|}{ Naturaleza } & \multicolumn{2}{c|}{$\begin{array}{c}\text { Artesanía y } \\
\text { folclore }\end{array}$} & \multicolumn{2}{c|}{ Gastronomía } & \multicolumn{2}{c|}{ Tranquil. } & \multicolumn{2}{c|}{$\begin{array}{c}\text { Eventos } \\
\text { culturales }\end{array}$} \\
\hline $39 \%$ & $48 \%$ & $22 \%$ & $32 \%$ & $1 \%$ & $2 \%$ & $14 \%$ & $15 \%$ & $20 \%$ & $2 \%$ & $1 \%$ & $0 \%$ \\
\hline
\end{tabular}

Leyenda: 1. Edad; 2. Mercados emisores; 3. Canales de información; 4. Motivación del viaje Fuente: Pardellas, X. (2009) Estratexias turísticas das cidades do Eixo Atlántico. Vigo

Observamos comparativamente que a Chaves acuden más jóvenes que a Verín (un 24\% frente al $8 \%$ de menores de 30 años) y que el mercado emisor principal en los dos casos es el nacional, pero también más acusado en Chaves. Sin embargo, las diferencias mayores 
aparecen en la información previa que consultaron para decidir el viaje y en las motivaciones personales. Así como los visitantes a Chaves utilizaron más las tecnologías de internet para decidir su viaje (un 25\%) y presentan una elevada fidelidad (el 17\% habían hecho una visita anterior), los que estuvieron en Verín se guiaron mayoritariamente por las recomendaciones de amigos y familiares (un 72\%) y apenas un $8 \%$ usaron previamente la red informática.

Por su parte, aunque las respuestas muestran lógicas variaciones, la motivación para el viaje guarda en los dos municipios una notable correspondencia con los recursos y ofertas de ambos, muy centradas en el patrimonio histórico y en la naturaleza, lo que a su vez es consecuente con la promoción llevada a cabo desde las dos administraciones locales en sus portales web, donde esa oferta es la más destacada.

\section{DIAGNOSIS ELEMENTAL: BARRERAS Y FACILITADORES}

Las dos ciudades contaban previamente con planes estratégicos de desarrollo (Cámara de Comercio de Verín. Informe 2006; Cámara Municipal de Chaves. Plano de Accão 2015), en los que aparece un interesante diagnóstico de situación y sobre todo, datos y análisis referidos a sus principales recursos y actividades económicas. Con esos documentos y la información extraída de las entrevistas y mesas de trabajo con agentes locales, la investigación se centró en detectar los principales factores que facilitaban o dificultaban el proceso de construcción de la eurociudad desde la perspectiva y la hipótesis de que el turismo podía constituir un elemento relevante en la cooperación y en el conjunto del proceso.

En general, destacan tres tipos de factores positivos y dos negativos. Los más favorables pueden situarse en la geografía (el río Támega discurre perpendicular a la frontera y por lo tanto configura lazos de unión territorial), en el protagonismo de la sociedad civil y de la administración local (las asociaciones culturales ya venían realizando actividades conjuntas desde hacía varios años y el Eixo Atlántico -asociación de municipios de la eurorregiónmostró desde el inicio un apoyo total al proyecto), y en tercer lugar, en la concurrencia de inversiones estatales en infraestructuras viarias, que sitúan a los dos municipios en un punto de unión de vías de alta capacidad desde el sur de Portugal y el centro de la península.

En el lado negativo han de citarse las asimetrías administrativas y competenciales (el gobierno de la Xunta de Galicia tiene capacidad normativa y legislativa en muchos de los aspectos que atañen a este proyecto, que no existen en la Região Norte), y desde luego, la escasa experiencia asociativa empresarial con serios problemas para innovar y competir.

El resumen del diagnóstico se presenta en la siguiente tabla simplificada de facilitadores y barreras. 


\section{ACTIVIDAD TURÍSTICA}

\section{FACILITADORES}

- Territorio con uniformidad geológica y ausencia de frontera física. Curso del río Támega, perpendicular a la frontera, conformando un singular corredor natural y ambiental

- Existencia de espacios naturales en toda el área y de zonas verdes urbanas con potencial para proyectar una imagen de calidad ambiental

- Núcleos rurales en el entorno con un importante valor patrimonial, histórico y cultural que configuran una identidad común

- Estruturas de cooperación a nivel regional (Eixo Atlántico, Comunidad de Trabajo Galicia - Norte de Portugal, Comunidade Territorial de Cooperación do Támega), así como experiencias de cooperación entre las dos ciudades a través de Interreg IIIA (Centro de Interpretación Museística, rede cultural)

- Asociaciones culturales con una cierta dinámica de programación de actividades conjuntas, especialmente en artes plásticas y musicales (referencia para organizar otros eventos en el futuro)

- Considerable actividad económica en torno al termalismo (Chaves) y de aguas embotelladas (Verín), con importantes inversiones en los últimos años y proyectos de diversificación a medio plazo

- Infraestructuras de comunicación entre las áreas urbanas que favorecen su conectividad

- Localización geográfica dentro de vías de alta capacidad, facilitando la accesibilidad desde la península al área conjunta

- Oferta hotelera con potencial para el diseño y desarrollo de un destino de excelencia

- Relevancia del comercio y la restauración como factores de interrelación, con experiencias recientes de cooperación público - privada e iniciativas de innovación para crear un espacio comercial común

\section{BARRERAS}

- Asimetrías en el tratamiento del corredor del río Támega, que dificulta su protección, clasificación e incluso gestión (Red Natura en Verín y todavía solicitud de paisaje protegido en Chaves)

- Ausencia de un tratamiento integrado del río Támega como recurso turístico de ocio y naturaleza

- Planes no armonizados de saneamiento y depuración de aguas y tratamiento de residuos. Retraso de Verín en la incorporación a la Agenda 21

- Marcos legales, fiscales, tarifarios, administrativos y burocráticos diferenciados a cada lado de la frontera, que dificultan una relación fluída en la Eurociudad, tanto en el ejercicio de derechos de ciudadanía, como en las relaciones económico-empresariales

- Asimetrías en la dimensión territorial, urbana, económica, comercial y de dotación de equipamientos entre Chaves y Verín, que no favorecen la percepción de un reparto equitativo de los costes-beneficios derivados del proceso de construción de la Eurociudad

- Diferente legislación y normativas jurídicas de planeamiento territorial de aplicación en los dos municipios, que además tienen diferencias compentenciales (mayores en el lado portugués), lo que dificulta la ordenación de la Eurociudad como un único territorio y la protección del paisaje

- Inexistencia de una plataforma informática de la Eurociudad, que permitiría el acceso interactivo desde mercados emisores a una información integrada en relación con el área (consultas, reservas, compras)

- Insuficiente nivel de profesionalización, especialmente en los establecimientos de menor categoría y en general, déficit formativo para consolidar una oferta de calidad

- Limitada presencia de inversiones transfronterizas y de estructuras de cooperación estables entre las asociaciones empresariales de la zona, con escasa cultura de emprendimiento e innovación, lo que resta posibilidades para la realización de proyectos de mayor dimensión 


\section{DISEÑO ESTRATÉGICO DEL DESTINO}

Con este diagnóstico y recuperando el marco científico comentado en el segundo apartado, es preciso situar el diseño del proyecto dentro de un primer ámbito de decisiones que orienten el desarrollo de la cooperación en la eurociudad hacia un modelo de competitividad micro (relación horizontal entre empresas buscando la complementariedad en la producción e incremento de la eficiencia, basada en las sinergias por el uso de recursos comunes), agregado a una actuación macro generadora de externalidades positivas (la coordinación de normativas y el apoyo institucional serán los desafíos más importantes a medio plazo para el éxito del proyecto).

En el mismo sentido desde el ámbito turístico, utilizando las aportaciones citadas de Leiper (1995), Dredge (1999) y Bigné y López (2000), se consideró que el núcleo central de atracción debía ser el conjunto formado por el río Támega y los recursos termales, que permitían definir una imagen de marca con la denominación, «Eurociudad del agua», aprobada por los dos regidores locales. Dado que el río discurre en perpendicular a la frontera permite un recorrido circular de ida y vuelta con dos nodos básicos en los núcleos urbanos de las dos ciudades y varios nodos y complejos de atracción secundarios en los pueblos próximos, que concentran recursos históricos, etnográficos y paisajísticos, así como también en las fuentes termales.

El diseño estratégico de la eurociudad como destino turístico quedó así estructurado en dos grandes ejes, actuando simultáneamente como nexos de unión para las actividades y de reconfiguración de todo el territorio: «el Corredor Ecológico del Támega» y la «Ciudad de la Salud», que en común deben tender a conformar un «Destino Termal de Excelencia».

El concepto de corredor ecológico aparece en varios trabajos sobre el análisis de espacios rurales y urbanos (Montseny 2000, Gurrutxaga 2004), aunque la aportación de Alberdi (2009) profundiza en aspectos que interesan especialmente a este proyecto, como son la conectividad ecológica y las zonas de contacto, considerando el carácter transfronterizo de las actuaciones. En ese sentido, para la definición del Corredor Ecológico del Támega ha de tenerse en cuenta que en el territorio de la eurociudad Chaves-Verín encontramos, como ya fue comentado, un hecho geográfico singular en comparación con el resto de la frontera entre Galicia y el Norte de Portugal, dado que en lugar de un elemento natural que marcaba frontera como es el caso del río Miño en la llamada «raya húmeda», o de sierras montañosas en la «raya seca», aquí el río es perpendicular a la frontera e históricamente nunca sirvió para complementar la barrera administrativa, sino al contrario, para favorecer lazos de unión entre las dos ciudades.

Esta característica representa un rasgo diferenciador notablemente positivo y relevante y la idea del corredor ecológico, además de acentuar su papel básico de comunicación, servirá para garantizar la calidad del agua no solamente del propio Támega, sino la de todos los acuíferos de la zona que constituyen un importante recurso social y turístico y permitirá iniciar ya en el corto plazo las acciones necesarias para su declaración como Reserva de la Biosfera Transfronteriza.

Las principales líneas de acción a promover en el Corredor Ecológico del Támega presentan un contenido muy amplio dado que son también muy diversos los aspectos que influyen en el concepto de territorio sostenible y que por lo tanto, afectan a la calidad ambiental 
de un destino turístico, el tratamiento de las aguas superficiales y soterradas, el cuidado del biotopo, las fuentes de energía, el paisaje como elemento natural y cultural, etc. En consecuencia, las medidas propuestas pretenden recoger aquellos aspectos y convertirlos en compromisos sociales e institucionales para la configuración del destino, lo que tendrá como objetivo paralelo la calidad de vida de los residentes:

1. Mejorar la calidad del agua y de los ríos como clave de desarrollo (eso implica actuar sobre las diversas fuentes y manantiales de los dos municipios, realizando un inventario y un programa global de reutilización, a fin de asegurar el uso sostenible de este recurso).

2. Crear un sistema verde de calidad (que no se limitaría a las márgenes del río, sino al conjunto de los espacios naturales del entorno, incorporando los recursos patrimoniales, culturales y paisajísticos conectados en red y en continuo con las propias zonas verdes urbanas de las dos ciudades. Este sistema debería ir acompañado de rutas de senderismo, para bicicletas y actividades al aire libre, así como de una o varias aulas de naturaleza para los escolares).

3. Transformar ambientalmente la Eurociudad a través de una Agenda 21 compartida (actuación ya iniciada desde el Eixo Atlántico en 2006 y que supondría simplemente la extensión y remodelación de las acciones para trabajar con la óptica de un único conjunto urbano en los programas y acciones de tipo ambiental, como por ejemplo, la generalización del uso de energías renovables en los equipamientos sociales y edificios administrativos con un programa de ahorro energético a medio plazo).

4. Rehabilitar el medio rural y el medio urbano tradicional (teniendo en cuenta que ya fueron realizadas intervenciones en los edificios y espacios públicos con otros programas europeos desde 2002, el nuevo enfoque implicaría una actuación coordinada para llevar a cabo actuaciones comunes y con criterios similares en los núcleos rurales de los dos municipios, estableciendo así un plan temporalizado de recuperación y puesta en valor de espacios etnográficos, culturales y arquitectónicos, para consolidarlos como nodos de atracción turística de calidad).

A su vez, para definir la Ciudad de la Salud, y considerando la existencia de importantes recursos termales en los dos municipios, el concepto debería ampliarse hacia el exterior de los ámbitos tradicionales de la prevención y la atención sanitaria, porque, tanto el propio concepto, como las propuestas enfocadas desde la configuración de un destino turístico, sobrepasan extensamente la definición tradicional de salud, que se reorientaría así a crear un nuevo modelo de referencia con la integración de varios recursos que acentúan el concepto de bienestar. En todo caso, y siempre desde el objetivo genérico de un uso socialmente racional de los recursos, es obvio que los existentes en este territorio constituyen una importante fuente de oportunidades para generar una oferta común termal y de salud, con características singulares y de notable calidad.

Por tanto, las líneas de acción a promover en la Ciudad de la Salud no pueden perder de vista las directrices europeas para la mejora de la calidad de vida de los ciudadanos, que se dirigen claramente en este caso a conseguir por parte de las administraciones la mayor eficiencia en la gestión de los recursos sanitarios. Por otra parte, debe apuntarse que en 2005 se creó en los dos municipios un Grupo de Trabajo para agilizar y profundizar la cooperación 
entre facultativos y gestores de instituciones médicas de ambas ciudades, con el objetivo de mejorar la atención médica al conjunto de la población.

A esas actuaciones debe agregarse que el termalismo moderno aparece mucho más orientado al disfrute y al relax que a la idea decimonónica de curación de ciertas enfermedades y este cambio, integrado en el citado concepto más amplio de bienestar, se incluyó en el proyecto de eurociudad para conformar una definición actual y atractiva de salud, por lo que las medidas propuestas afectan igualmente a los residentes y a los turistas visitantes:

1. Garantizar el acceso universal a los servicios públicos de salud (para lo que se debe aprovechar el antes citado Grupo de Trabajo, estableciendo un protocolo oficial que permita el intercambio de personal y medios, teniendo en cuenta que existen evidencias de complementariedad entre los servicios y las prestaciones de los hospitales de cada ciudad y que hay antecedentes de cooperación anterior en actuaciones sanitarias de especial relevancia, lo que llevaría a una reestructuración geográfica y de agrupación de servicios para incrementar las tasas de cobertura en atención primaria de familia.)

2. Incrementar el valor social y de mercado de los recursos termales a través de la cooperación empresarial (las implicaciones de un destino termal moderno obligan a cambios en los objetivos empresariales, reorientando en ese sentido la estrategia de mercado, las inversiones y la propia actividad, desde la consideración de que los nuevos productos y servicios tendrán como destinatarios preferentes los segmentos de demanda de ingresos medio-altos, que acuden a estas instalaciones para compensar el estrés de su trabajo y que además están habituados a un nivel alto de calidad en los servicios. En paralelo y complementariamente, la creación de un Centro de Innovación en productos termales, tendería a acentuar la colaboración público-privada, por ejemplo, con personal científico de la universidad)

3. Convertir la Eurociudad en un referente europeo de salud, naturaleza y agua (en esa línea, se apunta la necesidad de reelaborar los requerimientos de uso de la tarjeta europea de salud para adaptarlos a este proyecto, marcando una especial atención a la formación profesional y superior del personal sanitario con aquel objetivo y diseñando un protocolo de coordinación entre la atención sanitaria general y la termal. Al mismo tiempo, integrando el corredor ecológico del Támega antes citado, con la creación de un Centro de Interpretación del Termalismo, completaría y singularizaría a este destino, ofreciendo a los clientes y a los visitantes-turistas no interesados específicamente en usar las instalaciones termales, una información y divulgación interesante sobre esta temática, que llegaría a convertirse en sí mismo en un producto turístico de referencia)

En última instancia, el objetivo de las citadas medidas será convertir a la eurociudad (ya bajo la denominación de Eurociudad del Agua), en un Destino Termal de Excelencia, centrado en la salud, la naturaleza y el ocio. Lógicamente, la consolidación de un espacio de excelencia turística termal en esta área requiere una visión integrada: una oferta innovadora y de calidad en los equipamientos y servicios especializados (balnearios), una oferta profesional en el campo de la hostelería y la restauración, un desarrollo complementario en servicios y actividades que dinamicen y generen sinergias (centro de interpretación del termalismo, 
productos de higiene y belleza, actividades comerciales, culturales y de ocio) y en todo caso, un énfasis especial en las ofertas turísticas existentes que ya fueron citadas (la ruta del Oro y las Vistas Mágicas, la ruta de los Embalses (Barragems), las rutas del Arte Rupestre y del Contrabando, además de la ramificación del Camino de Santiago desde el Algarve, entre otras). Todo ello unido a la construcción de un entorno amistoso, saludable y ambientalmente sostenible.

\section{EVALUACIÓN EX ANTE Y CONCLUSIONES}

Considerando las informaciones derivadas de las reuniones con los agentes sociales, económicos e institucionales implicados en el proyecto de la eurociudad, se puede deducir que la percepción de los actores locales es altamente positiva con respecto a las posibilidades de llevarlo a cabo, sobre todo si tenemos en cuenta los antecedentes de cooperación en algunos casos concretos (sanidad, educación, cultura), que deben ser aprovechados ya en el corto plazo, así como el aparente completo apoyo de las instituciones supramunicipales (Xunta de Galicia y Comisão de Coordenação e Desenvolvimento da Região Norte, como ya fue comentado en la introducción de este documento).

Es interesante en todo caso, construir un esquema de previsión de efectos a medio plazo, teniendo en cuenta en primer lugar que las propuestas planteadas han sido aceptadas y asumidas por los principales actores sociales e institucionales del territorio, y en segundo lugar, que como afirman Pizam y Mansfeld (2000), el turismo está funcionando como un factor de desarrollo urbano y territorial, generando importantes modificaciones en el sistema de asentamientos y en la dotación de infraestructuras y equipamientos, lo que actúa como un elemento favorecedor de nuevas oportunidades de emprendimiento y de creación de empleo.

Hemos establecido tres fuentes de impacto, ambientales, socioculturales y económicas, siguiendo las aportaciones de Brunet y Belzunegui (1999), actualizadas por Vera e Ivars (2003), para los efectos ambientales, de Sastre y Payeras (2002), para los socioculturales y de Goeldner y Ritchie (2000) y Martinez Roger (2003), para los efectos económicos. Ese análisis fue también aplicado por los autores al municipio de Caldas de Reis con un esquema similar (Pardellas 2004):

\section{IMPACTOS AMBIENTALES}

Características situación actual

- Los problemas en el saneamiento y depuración de las aguas vertidas al río Támega reducen su atractivo turístico

- Las asimetrías en la gestión de los espacios naturales en torno al río Támega generan decisiones descoordinadas e ineficientes

- Unos recursos naturales infrautilizados suponen una pérdida de oportunidades de creación de nuevas actividades y empresas turísticas, así como de empleo y riqueza

\section{Consecuencias estimadas del modelo}

- La propuesta de una Agenda 21 compartida tenderá a transformar la óptica de las actuaciones hacia un único conjunto territorial, generalizando el objetivo común de conseguir la excelencia en la calidad de las aguas

- La actuación dirigida a elaborar un tratamiento integral de los espacios naturales y urbanos, debe suponer un objetivo de reconfiguración del territorio en torno a una Red Natura conjunta, estableciendo controles estándar en los dos municipios que forman la Eurociudad 


\section{IMPACTOS AMBIENTALES}

\section{Características situación actual}

- Existe ahora mismo un riesgo de colonización de espacios naturales para urbanizar, teniendo en cuenta la demanda de $2^{a}$ residencia en esta comarca

\section{Consecuencias estimadas del modelo}

- La propuesta de crear un Sistema Verde deCalidad debe extender la gestión turística de la naturaleza, desbordando los límites del río (que en todo caso, mantendría su papel de nodo atractivo central) y propiciando la elaboración de ofertas de turismo activo, con rutas circulares que permitan conocer todo el territorio desde la filosofía común de su sostenibilidad

- La nueva configuración territorial y administrativa debe implicar una normativa común de usos del suelo, estableciendo espacios de urbanización regulados para evitar la degradación del paisaje

\section{IMPACTOS SOCIOCULTURALES}

\section{Características situación actual}

- Los problemas administrativos dificultan el aprovechamiento de las estructuras de cooperación que aporta la existencia de la asociación de municipios Eixo Atlántico

- Las diferentes legislaciones constituyen una fuerte barrera para utilizar adecuadamente los servicios técnicos y humanos de salud, a pesar de su notable complementariedad actual
Consecuencias estimadas del modelo

- Las actuaciones para la homologación de servicios y de formación profesional deben influir no solo en el aumento de la cohesión y la equidad territorial, sino también en la diversificación y dinámica de las relaciones culturales, facilitadas por el idioma común, lo que favorece al mismo tiempo una identificación de las dos comunidades sociales con un mismo proyecto vital y cultural de futuro a largo plazo. Esto debe ayudar a la creación de nuevos paisajes culturales en este territorio

- El objetivo de garantizar un acceso universal a los servicios públicos de salud para los residentes en los dos municipios está sustentado en la experiencia del Grupo de Trabajo de Salud, que ya estableció las bases de los principales protocolos de cooperación, lo que con la desaparición de las barreras legislativas (por ejemplo, de circulación transfronteriza de ambulancias para el traslado de enfermos), debe favorecer la transformación de la Eurociudad en una referencia europea de salud 


\section{IMPACTOS ECONÓMICOS}

Características situación actual

- La falta de tradición y la escasez de estructuras de cooperación empresarial, tanto interna, como transfronteriza, reduce las oportunidades de innovación y sobre todo, la competitividad territorial en relación, por ejemplo, a las villas y ciudades integradas en el eje litoral de la eurorregión y desde luego, con respecto al resto de la península y a Europa

- A pesar de su complementariedad objetiva, las empresas que explotan los recursos termales (agua embotellada en Verín, usos balnearios en Chaves), compiten entre si y apenas toman decisiones conjuntas para la mejora de sus ofertas

\section{Consecuencias estimadas del modelo}

- Las actuaciones macro para fomentar la cooperación deben tener ya en el corto plazo, un efecto micro de innovación y creación de empresas y empleo, ante la perspectiva de un nuevo y económicamente más extenso e interesante mercado, tanto para el comercio, como para la ya citada actividad turística

- Aún admitiendo las dificultades para lograr el objetivo de consolidar un destino termal de excelencia, la dinámica de superación de la calidad y de innovación que deben poner en marcha las actuaciones de fomento de la cooperación empresarial en el proceso de construcción de la Eurociudad, podría suponer en el medio plazo un notable incremento del prestigio de las instalaciones balnearias, al tiempo que una creación neta de empleo, influyendo en el mismo sentido en los servicios complementarios

Como elementales conclusiones pueden destacarse:

- El territorio conjunto cuenta con recursos -algunos de ellos ya convertidos en productos y ofertas actuales-, que le permiten elaborar una oferta turística coordinada y atractiva para los mercados emisores de la península y Europa.

- El río Támega aparece como núcleo central del destino denominado Eurociudad del agua, por su situación geográfica y su discurrir norte-sur, perpendicular a la frontera, que lo convierte en el principal factor de remodelación del territorio. A la par, los recursos termales constituyen el segundo elemento genérico que sirve para identificar la singularidad del destino.

- Las dos ciudades ocupan el lugar de núcleos básicos de configuración del destino termal, con potencialidad de excelencia, con nodos de atracción secundarios (sobre la base del paisaje y la cultura), para los segmentos de demanda interesados en este tipo de ofertas. Cada una de ellas presenta además recursos que complementan a la vecina, por lo que objetivamente, es razonable imaginar en el medio plazo una nueva estructura económica de su sistema productivo que pueda ser presentada y promocionada como un referente dentro del termalismo moderno europeo.

- El objetivo último de las actuaciones propuestas es conseguir la mayor cohesión territorial posible, lo que unido a los antecedentes y prácticas de cooperación observados, estaría favoreciendo la construcción de un nuevo paisaje cultural, fundamentando en el idioma común y en el elevado grado de aceptación social del proyecto de eurociudad.

- Agregadamente, el notable apoyo institucional a aquel proyecto permite imaginar igualmente un escenario de reducción e incluso de eliminación en ciertos casos, de las barreras y obstáculos derivados de las asimetrías en las competencias administrativas de las dos regiones. 
- El principal punto débil sin embargo, radica en la aparente falta de cultura de colaboración empresarial en las actividades turísticas y más en concreto en los apartados referidos al termalismo, imprescindible para elaborar cualquier proyecto de uso conjunto de los recursos y sin la que no es posible promocionar la eurociudad como destino termal de excelencia.

\section{REFERENCIAS BIBLIOGRÁFICAS}

ALBERDI, J.C. (2009): «Paisaje, capacidad agrológica y conectividad en la planificación municipal. Desarrollo de una aplicación (Andoain-Guipúzcoa)», Boletín de la Asociación de Geógrafos Españoles, no ${ }^{\circ}$ 0, pp. 239-258.

AYDALOT, P. (1986): Milieux innovateurs en Europe. Prensa Económica. París.

BEGG, I. (1999): «Cities and Competitiveness», Urban Studies, volume 36, n 5/6, pp. 795 809.

BIGNÉ, J. E. y LÓPEZ, D. (Dir.) (2000): Planificación territorial y comercialización turística. Serv. Pub. Univ Jaume I. Castellón.

CAMAGNI, R. (2002): «On the concept of territorial competitiveness; sound of misleading», Urban Studies, vol. $39 \mathrm{n}^{\circ} 13$.

CCDRN (2007): Política e orentaçôes estratégicas para o desenvolvemento turístico da Regiâo Norte. Doc. Preliminar. Porto.

CUEVAS, T y ZIZALDRA, I. (2009): «Red transfronteriza en turismo. Formación gestora caso Ciudad Juárez, Chihuahua y El Paso, Texas, Estados Unidos de Norteamérica» Revista de Análisis Turístico, $\mathrm{n}^{\mathrm{0}}$ 7, pp. 22-38.

DOMINGUEZ, L. (Dir.) (2008): Chaves-Verín: a eurocidade da auga. Ed. Eixo Atlántico. Vigo.

DREDGE, D. (1999): «Planificación y Diseño de Destinos Turísticos». Annals of Tourism Research en Español 2 (1), pp. 394-414.

ECORYS (2003) A study on the factors os regional competitiveness. Rotterdam.

FERNÁNDEZ TABALES, A., et al (2009): «El concepto de cohesión territorial. Escalas de aplicación, sistemas de medición y políticas derivadas», Boletín de la Asociación de Geógrafos Españoles, n ${ }^{\circ}$ 50, pp. 157-172.

FIGUEIREDO, A. y PARDELLAS, X. (2005): «Innovaçâo e competitividade nas cidades do Eixo Atlántico e as súas áreas de influencia» in Souto, XM et al. Segundos Estudos Estratéxicos do Eixo Atlántico. Ed. Eixo Atlántico. Vigo.

FIGUEIREDO, A y SILVA, M.R. (2004): «Factores territoriais e institucionais de internacionalizaçâo da cidade-regiâo» in Actas Congreso Porto Cidade-Regiâo. Serv. Pub Univ Porto.

GAROFOLI, G. (1983): «Le aree sistema in Italia», Politica e Economía, vol. 11, pp. 17-34. GAROFOLI, G. (1992): Endogenous development and southern Europe. Avebury. Aldeshot GOELDNER, C.; RITCHIE, B. y MC INTOSH, W. (2000): Tourism principles, practices. Wiley \& Sons. New York.

GONZÁLEZ, J. y FIGUEROA, P. (2005): Plan Estratégico de Innovación de Galicia 2010. Xunta de Galicia. Consellería de Innovación, Industria e Comercio. Dirección Xeral de I+D. A Coruña. 
GONZÁLEZ, J. y FIGUEROA, P. (2006): Visión estratégica del Sistema Empresarial del Área Metropolitana de Vigo. Evolución 2000-2005 y expectativas. Instituto de Desarrollo Caixanova. Vigo.

GONZÁLEZ, J. y FIGUEROA, P. (2007): Visión Estratégica del Sistema Productivo del Área Metropolitana de A Coruña. Instituto de Desarrollo Caixanova. Vigo.

GONZÁLEZ, J. y FIGUEROA, P. (2008): Visión Estratégica del Sistema Productivo de las comarcas de Ferrol, Eume y Ortegal. Instituto de Desarrollo Caixanova. Vigo.

GUNN, G.A. (1972): Vacationscape: Designing Tourist Regionals. Austin: University of Texas.

GUNN, G.A. (1988): Tourism Planing.New York: Taylor and Francis.

GUNN, G.A. (1993): Tourism Planning: basics, concepts, cases. London: Taylor\& Francis.

GURRUTXAGA, M. (2004): Conectividad ecológica del territorio y conservación de la biodiversidad. Nuevas perspectivas en ecología del paisaje y ordenación territorial. Informe técnico $n^{\circ} 103$. Servicio Central de Publicaciones del Gobierno Vasco. Vitoria-Gasteiz.

KRUGMAN, P. (1994): «Competitiveness - a dangerous obsession», Foreign Affairs, volume 73, pp. 28-44.

KRUGMAN, P. (1996): «Making sense of the competitiveness debate», Oxford Review of Economic Policy, volume 12, pp. 17-35.

LEIPER, N. (1995): Tourism Management. Melbourne: RMIT Press.

LUE, C., CROMPTON, J. y FESENMAIER (1993): «Conceptualization of Multidestination Pleasure Trips». Annals Of Tourism Research, vol. 20, pp. 289-301.

MARTINEZ ROGER, F. (2003): Turismo rural: Características de la actividad e impacto económico en Galicia. (Tesis Doctoral). Santiago de Compostela.

MONTSENY, A. (2000): «Paisatge, espais naturals i turisme». Revista de Debats Terrritorials, $\mathrm{n}^{\circ} 8$, pp. 139-144.

OPPERMAN, M. (1993) «Tourism Space in Developing Countries». Annals of Tourism Research, vol. 20, pp. 535-560.

PARDELLAS, X. (2004): «Una propuesta de turismo sostenible para el municipio de Caldas de Reis», Cuadenos de Turismo, nº 13, pp. 107-126.

PARDELLAS, X. (2009): Estratexias turísticas das cidades do Eixo Atlántico. Ed. Eixo Atlántico. Vigo.

PEARCE, D. (1995): Tourism Today: A Geographical Análisis ( 2nd ed.). New York: Longman.

PIZAM A. y MANSFELD Y. (Ed.) (2000): Consumer Behavoir in Travel and Tourism. Haworth press. Binghamton.

PORTER, M.E. (2002): «Building the microeconomic foundations of prosperity: Findings from the microeconomic competitiveness index», in The Global Competitiveness Report 2002.

PORTER, M.E. (1990 y 1998): The Competitive Advantage of Nations, London: Mac Millan

SALA MARTIN, X. (2003): Executive Summary of the Global Competitiveness Report 2003, World Economic Forum.

SASTRE, A. y PALLERAS, M. (2002): «Impactos económicos, sociales y medioambientales del turismo en las islas Baleares». XXVIII Reunión Estudios Regionales. Murcia. 
SWARBROOKE, J., HORNER, S. (1999): Consumer Behavior in Tourism. Oxford: Butterworth Heinemann

TUROK, I. (2004): «Cities, Regions and Competitiveness», Regional Studies, volume 38, $\mathrm{n}^{\circ} 9$.

VALLS, J.F. (2004): Gestión de destinos turísticos sostenibles. Ed. Gestión 2000. Madrid.

VÁZQUEZ BARQUERO, A. (1983): «Industrialization in rural areas. The Spanish case», in $C T / R U R / 113 / 06, O C D E$.

VÁZQUEZ BARQUERO, A. (1999): Desarrollo, redes e innovación. Pirámide. Madrid.

VÁZQUEZ BARQUERO, A. (2005): Las nuevas fuerzas del desarrollo. A. Bosch Ed. Barcelona.

VERA, F e IVARS, J.A. (2003): «Sistema de indicadores aplicado a la planificación y gestión del desarrollo turístico sostenible», in Valdés L. Experiencias públicas y privadas en el desarrollo de un modelo turístico sostenible. Fundación Universidad de Oviedo. 
